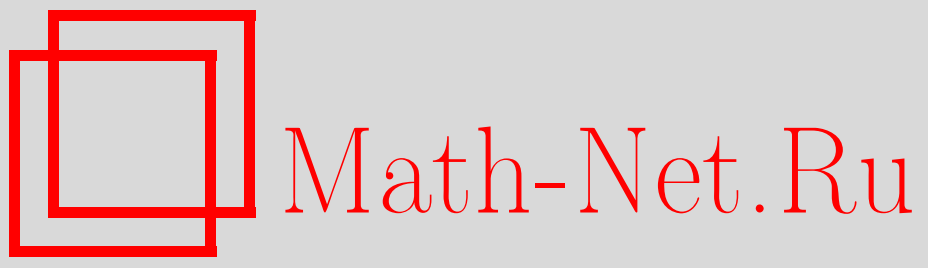

М. Ю. Иванов, О связи задач максимизации степенной и логарифмической полезности в экспоненциальной модели Леви, Теория вероятн. и ее примен., 2014, том 59, выпуск 4, 781-790

DOI: https://doi.org/10.4213/tvp4596

Использование Общероссийского математического портала Math-Net.Ru подразумевает, что вы прочитали и согласны с пользовательским соглашением http://www . mathnet.ru/rus/agreement

Параметры загрузки:

IP : 54.197 .217 .227

26 апреля 2023 г., 14:50:50

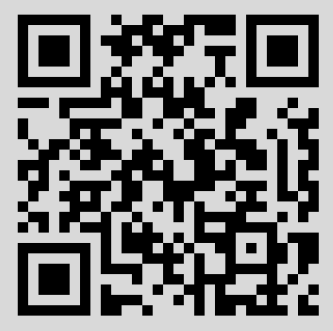


7. Беломестный Д. В., Прохоров А.В. О задаче восстановления генерального распределения по распределению линейной статистики. - Вестн. Моск. ун-та. Сер. мат.-мех., 2003, в. 2, с. 3-8.

8. Лоэв М. Теория вероятностей. М.: ИЛ, 1962, 277 с.

9. Садикова С. М. Двумерные аналоги неравенства Эссеена с применением к центральной предельной теореме. - Теория вероятн. и ее примен., 1966, т. 11, в. 3, c. $369-380$.

Поступила в редакцию

9.II.2013

Исправленный вариант

10.III. 2014

(c) $2014 \Gamma$.

ИВАНОВ М. Ю.*

\title{
О СВЯЗИ ЗАДАЧ МАКСИМИЗАЦИИ СТЕПЕННОЙ И ЛОГАРИФМИЧЕСКОЙ ПОЛЕЗНОСТИ В ЭКСПОНЕНЦИАЛЬНОЙ МОДЕЛИ ЛЕВИ
}

\begin{abstract}
В данной статье мы решаем задачу максимизации степенной полезности в экспоненциальной модели Леви путем сведения ее к задаче максимизации логарифмической полезности (точнее, к задаче нахождения эталонного портфеля) в той же модели, но с другим процессом Леви. Также получена связь между решениями двойственных задач.
\end{abstract}

Ключевые слова и фразы: степенная полезность, логарифмическая полезность, экспоненциальная модель Леви.

1. Введение и основные результаты. В данной работе мы рассматриваем задачу, в которой агент на финансовом рынке пытается с помощью выбора правильной стратегии поведения максимизировать ожидаемую полезность своего портфеля в конечный момент времени. Эта проблема достаточно много исследовалась в финансовой математике. В случае, когда множество мартингальных мер состоит из одной-единственной меры и рынок является полным, задача была решена, к примеру, в работе [10]. Д. Крамков и В. Шахермайер в [12] исследовали вопрос максимизации ожидаемой полезности в общей модели неполных рынков, где цены активов являются семимартингалами. Вместе с исходной проблемой решалась и двойственная задача, были найдены различные соотношения между решениями этих двух задач.

Одной из наиболее популярных моделей для неполных рынков является экспоненциальная модель Леви. Некоторые результаты для нее были получены, например, в работах [2], [4], [9], [11], [14].

Цель данной статьи состоит в решении задачи максимизации степенной полезности и двойственной к ней в общей экспоненциальной модели Леви при помощи результатов о максимизации логарифмической полезности из [5]. Исследуется связь между этими двумя случаями, из которой вытекают и другие интересные следствия.

Введем модель семимартингального рынка с одним активом, следуя известным работам [1], [9], [12]. На вероятностном пространстве $(\Omega, \mathscr{F}, \mathbb{F}, P)$, где фильтрация $\mathbb{F}=\left(\mathscr{F}_{t}\right)_{0 \leqslant t \leqslant T}$ непрерывна справа, рассмотрим семимартингал $S$ с действительными неотрицательными значениями, у которого траектории непрерывны справа и имеют предел слева на интервале времени $[0, T]$. Он моделирует дисконтированную цену

* Московский государственный университет им. М.В. Ломоносова, Москва, Россия; e-mail: m_y_ivanov@mail.ru 
актива. Введем самофинансируемый портфель П как пару $(x, H)$, где константа $x$ означает начальный капитал портфеля, а $H=\left\{H_{t}\right\}$ - предсказуемый процесс, интегрируемый по $S$. Мы интерпретируем $H_{t}$ как количество единиц актива в портфеле в момент $t$. Процесс капитала $X=\left(X_{t}\right)_{0 \leqslant t \leqslant T}$ такого портфеля П будет следующим:

$$
X_{t}=x+\int_{0}^{t} H_{u} d S_{u}, \quad 0 \leqslant t \leqslant T .
$$

Пусть $\mathscr{X}(x)$ обозначает семейство процессов капиталов с начальным значением $x$, которые в каждый момент времени неотрицательны:

$$
\mathscr{X}(x)=\left\{X_{t} \geqslant 0: X_{0}=x\right\} .
$$

В дальнейшем полагаем $\mathscr{X}=\mathscr{X}(1)$. Соответствующий процесс $H$ называют стратегией.

На нашем рынке присутствует агент, цель которого - максимизировать ожидаемую полезность капитала в конечный момент времени для степенной функции $U(x)=x^{p} / p, p<1, p \neq 0$ :

$$
u(x)=\sup _{X \in \mathscr{X}(x)} E U\left(X_{T}\right) .
$$

В дальнейшем мы будем выделять два случая для $p$ - когда $p<0$ и когда $0<p<1$. Последний случай является более сложным, многие соотношения и выкладки для $0<p<1$ будут очевидным образом выполняться для $p<0$. Также отметим, что для $p<0$ функция $U(x)$ отрицательна и, как следствие, всегда ограничена сверху. Так как $\mathscr{X}(x)=x \mathscr{X}$, то задачу достаточно решить для $x=1$. В наших рассуждениях мы будем исходить из предположения, что $u(x)<\infty$, равносильного тому, что решение $X^{*}$ задачи (2) удовлетворяет соотношению $E\left(X_{T}^{*}\right)^{p}<\infty$. Двойственная к (2) задача имеет вид

$$
v(y)=\inf _{Y \in \mathscr{Y}(y)} E V\left(Y_{T}\right)
$$

где $V(y)=\sup _{x>0}(U(x)-x y), y>0$, а $\mathscr{Y}(y)=\left\{Y_{t}>0: Y_{0}=y,\left(X_{t} Y_{t}\right)\right.$ есть супермартингал для любого $X \in \mathscr{X}, 0 \leqslant t \leqslant T\}$. Отметим, что если $U(x)=x^{p} / p, p<1, p \neq 0$, то $V(y)=((1-p) / p) y^{p /(p-1)}$. Поскольку $\mathscr{Y}(y)=y \mathscr{Y}(1)$, то двойственную задачу (3) достаточно решить для $y=1$ в рассматриваемом случае.

В работе предполагается, что цена актива есть стохастическая экспонента процесса Леви $L$ :

$$
S=\mathscr{E}(L), \quad \text { где } \Delta L>-1 .
$$

Эта модель называется экспоненциальной моделью Леви. Любой процесс Леви однозначно определяется триплетом своих характеристик $\left(B, C, \nu_{L}\right)$, в одномерном случае он имеет вид

$$
B_{t}(\omega)=b t, \quad C_{t}(\omega)=c t, \quad \nu_{L}(\omega, d t, d x)=d t \nu(d x),
$$

где константы $b, c(c \geqslant 0)$ и мера $\nu$ такая, что $\int\left(1 \wedge x^{2}\right) d \nu<\infty$, однозначно определяются из представления Леви-Хинчина характеристической функции:

$$
E e^{\imath u L_{t}}=\exp \left\{t\left(b u-\frac{1}{2} c u^{2}+\int\left(e^{\imath u x}-1-\imath u h(x)\right) d \nu\right)\right\}
$$

(мы полагаем $h(x)=x \mathbb{I}_{|x| \leqslant 1}$ ). В дальнейших рассуждениях триплет характеристик называем просто триплетом и пишем его в сокращенном виде без времени - $(b, c, \nu)$. Исключим из рассмотрения тривиальный случай, когда $L=0$.

Напомним, что процесс $L$ является монотонным тогда и только тогда, когда либо $c=0, \nu[x<0]=0, b-\int x \mathbb{I}_{|x| \leqslant 1} \nu(d x) \geqslant 0$, либо $c=0, \nu[x>0]=0$, $b-\int x \mathbb{I}_{|x| \leqslant 1} \nu(d x) \leqslant 0$ (см. [15]). Если процесс $L$ является монотонным (и ненулевым), то $u(x) \equiv+\infty$ как для логарифмической полезности, так и для степенной при 
$0<p<1$ (при $p<0$ выполнено равенство $u(x) \equiv 0$ ). Поэтому мы также исключаем этот случай из дальнейшего рассмотрения. Отметим, что в экспоненциальной модели Леви немонотонность процесса $L$ эквивалентна всем условиям типа отсутствия арбитража (см. [11]).

Задача (2) для логарифмической функции полезности исследовалась во многих работах (к примеру, в [9], [3]). Нам будет удобно понимать под задачей максимизации логарифмической полезности несколько более общую задачу, а именно задачу отыскания эталонного портфеля (см. определение 1 ниже). Хорошо известно, что портфель, максимизирующий логарифмическую полезность, всегда является эталонным, но возможна ситуация, когда портфель существует, а ожидаемая логарифмическая полезность равна $+\infty$. В нашей статье [5] для экспоненциальной модели Леви указаны вид эталонного портфеля и необходимые и достаточные условия для того, чтобы он был решением задачи максимизации логарифмической полезности.

О п р е д е л е н и е 1 . Портфель $\widetilde{X} \in \mathscr{X}$ называется эталонным, если $P\left(\inf _{t} \widetilde{X}_{t}>0\right)=1$ и для любого $X \in \mathscr{X}$ отношение $X / \widetilde{X}$ является супермартингалом.

В общей конечномерной семимартингальной модели рынка достаточно часто оптимальный портфель является эталонным по некоторой эквивалентной мере. $\mathrm{K}$ примеру, из рассуждений работы [16] следует, что если выполнено так называемое условие отсутствия неограниченной прибыли с ограниченным риском (NUPBR) и терминальное значение капитала портфеля строго положительно и является максимальным элементом среди терминальных значений процессов из класса $\mathscr{X}$, то этот портфель является эталонным по некоторой эквивалентной мере.

Если предположить, что в задаче максимизации полезности для функции $U$ выполнены условия теоремы 2.2 в [12], то можно указать явный вид меры $Q$ (относительно $P$ ), по которой портфель $X^{*}(x)$ для $U$ будет эталонным:

$$
\frac{d Q}{d P}=\frac{X_{T}^{*}(x) U^{\prime}\left(X_{T}^{*}(x)\right)}{x u^{\prime}(x)}=\frac{Y_{T}^{*}\left(u^{\prime}(x)\right) I\left(Y_{T}^{*}\left(u^{\prime}(x)\right)\right)}{x u^{\prime}(x)}, \quad I=-V^{\prime} .
$$

Действительно, по этой теореме терминальные значения решений прямой и двойственной задачи $X^{*}$ и $Y^{*}$ связаны соотношениями $X_{T}^{*}(x)=I\left(Y_{T}^{*}\left(u^{\prime}(x)\right)\right), Y_{T}^{*}\left(u^{\prime}(x)\right)=$ $U^{\prime}\left(X_{T}^{*}(x)\right)$, где $I$ есть обратная функция к $U^{\prime}$, а процесс $X^{*}(x) Y^{*}\left(u^{\prime}(x)\right)$ является равномерно интегрируемым мартингалом на $[0, T]$. Это означает, что мы можем по формуле (5) корректно задать меру $Q$, для которой $Z=X^{*}(x) Y^{*}\left(u^{\prime}(x)\right) /\left(x u^{\prime}(x)\right)$ есть процесс плотности. По определению $Y^{*}$ для любого $X \in \mathscr{X}$ процесс $X Y^{*}$ является супермартингалом, тогда $\left(X / X^{*}\right) Z$ также есть супермартингал по $P$, а $X / X^{*}$ - по $Q$. Значит, $X^{*}$ является эталонным портфелем по $Q$, что и требовалось показать.

Из формулы (5) следует, что для нахождения $Z$ необходимо знать решение либо прямой, либо двойственной задачи. В данной работе для экспоненциальной модели Леви и степенной полезности мы укажем явный вид $Z$ и $Q$ в терминах триплета $(b, c, \nu)$. Оказывается, по мере $Q$ процесс $L$ также является процессом Леви. Таким образом, задача максимизации степенной полезности сводится к задаче нахождения эталонного портфеля в экспоненциальной модели Леви. Также отметим, что для степенной полезности выполнены условия вышеупомянутой теоремы, процесс плотности $Z$ не зависит от $x$, так как $\mathscr{X}(x)=x \mathscr{X}$ и $\mathscr{Y}(y)=y \mathscr{Y}(1)$, а формула (5) приобретает вид

$$
\frac{d Q}{d P}=\frac{\left(X_{T}^{*}(1)\right)^{p}}{u^{\prime}(1)}=\frac{\left(Y_{T}^{*}\left(u^{\prime}(1)\right)\right)^{p /(p-1)}}{u^{\prime}(1)} .
$$

Задача максимизации степенной полезности рассматривалась в различных статьях. K примеру, в [9] она была решена в многомерном случае при наличии некоторых предположений, одно из которых по сути означало, что решение двойственной задачи есть эквивалентная мартингальная мера. В работе [8] авторы исследовали 
двойственную задачу в этом же предположении. У нас же в статье задача решается без этого предположения. В статье [13] основная задача была решена при помощи двойственной, при этом предполагалось отсутствие скачков у процесса цены акций. В [14] данная проблема исследовалась при наличии процесса потребления и многомерном процессе цены акций. Был найден определенный вид решения основной задачи, потом делался переход к двойственной задаче, для которой в свою очередь также находился некоторый вид решения в предположении его существования.

В данной работе мы рассматриваем максимально общую ситуацию в одномерном случае. Никаких ограничений на $L$ не накладывается, кроме необходимого предположения об отсутствии арбитража. Основная наша цель - показать, что оптимальный портфель в задаче степенной полезности всегда является эталонным портфелем относительно некоторой эквивалентной меры, по которой процесс $L$ также является процессом Леви. При этом и искомый портфель, и эта мера полностью определяются триплетом Леви процесса $L$. Помимо этого, подобная связь имеет место и для решения двойственных задач. С ее помощью нетрудно записать аналог теоремы 1 в [5], т.е. классифицировать в терминах процесса $L$ случаи, характеризующие мартингальные свойства решения двойственной задачи.

Следующая теорема показывает связь между решениями задач максимизации степенной полезности и логарифмической.

Теорема 1. Пусть дана задача максимизачии степенной полезности (2) для мерь $P$, относительно которой $L$ является немонотонньм прочессом Леви. Тогда условие $E\left(X_{T}^{*}\right)^{p}<\infty$ эквивалентно $\int_{x>1} x^{p} d \nu<\infty$. В этом предположении существует единственная константа $y^{*}$, которая задает такую меру $Q$ с параметрами Гирсанова $(\beta, Y)=\left(y^{*},\left(1+y^{*} x\right)^{p}\right)$, что по ней $\mathscr{E}\left(y^{*} L\right)$ является эталонньм портфелем. При этом $\mathscr{E}\left(y^{*} L\right)$ является оптимальным портфелем и по исходной мере $P$ для задачи степенной полезности.

Решение, когда оно существует, в степенном и логарифмическом случае всегда имеет вид $\mathscr{E}\left(y^{*} L\right)$, где $y^{*}$ есть константа, полностью определяемая триплетом $L$. В дальнейшем под решением иногда будем подразумевать именно эту константу. Также отметим, что для случая $p<0$ условие $E\left(X_{T}^{*}\right)^{p}<\infty$ выполнено ввиду того, что в этом случае в $(2) U(x)<0$; при $p<0$ выполнено и условие $\int_{x>1} x^{p} d \nu<\infty-$ по определению процесса Леви; эквивалентность этих условий очевидна.

С помощью теоремы III.3.24 в [7] для процесса $L$ нетрудно посчитать триплет характеристик $\left(B^{\prime}, C^{\prime}, \nu_{L}^{\prime}\right)$ относительно меры $Q$ из теоремы 1 . Он имеет вид

$$
B_{t}^{\prime}=b^{\prime} t, \quad C_{t}^{\prime}=c^{\prime} t, \quad \nu_{L}^{\prime}(\omega, d t, d x)=d t \nu^{\prime}(d x),
$$

где

$$
\begin{aligned}
& b^{\prime}=b+c p y^{*}+h(x)\left(\left(1+y^{*} x\right)^{p}-1\right) * \nu, \\
& c^{\prime}=c \\
& \nu^{\prime}=Y \nu=\left(1+y^{*} x\right)^{p} \nu
\end{aligned}
$$

т.е. является детерминированным и однородным. Таким образом, $L$ остается процессом Леви по новой мере $Q$.

Теорема 1 ставит в соответствие каждой задаче максимизации степенной полезности в экспоненциальной модели Леви задачу нахождения эталонного портфеля в другой экспоненциальной модели Леви. Здесь возникает вопрос: является ли данное отображение взаимно однозначным? Следующее утверждение дает положительный ответ и дополняет теорему 1. Для формулировки результата введем дополнительные обозначения. Пусть $\mathscr{Q}$ есть множество триплетов, задающих немонотонный процесс Леви, а $\mathscr{Q}_{p}$ есть такое подмножество $\mathscr{Q}$, что для триплета выполнено $\int_{x>1} x^{p} d \nu<\infty$. Для случая $p<0$, как нетрудно понять, $\mathscr{Q}_{p}$ совпадает с $\mathscr{Q}$. 
Теорема 2. Отображение из теоремь 1, которое триплету $(b, c, \nu)$ из $\mathscr{Q}_{p}$ по формулам (6) ставит в соответствие триплет $\left(b^{\prime}, c^{\prime}, \nu^{\prime}\right)$ из $\mathscr{Q}$, является взаимно однозначным между $\mathscr{Q}_{p}$ и $\mathscr{Q}$.

Напомним [5, теорема 1], что для процесса $1 / \mathscr{E}\left(y^{*} L\right)$ по мере $Q$ существуют 3 возможности:

1) $1 / \mathscr{E}\left(y^{*} L\right)$ есть процесс плотности эквивалентной мартингальной меры относительно $Q$;

2) $1 / \mathscr{E}\left(y^{*} L\right)$ является мартингалом, но не является эквивалентной $\sigma$-мартингальной плотностью;

3) $1 / \mathscr{E}\left(y^{*} L\right)$ есть супермартингал, но не локальный мартингал по мере $Q$.

Классификация этих трех случае в терминах триплета $\left(b^{\prime}, c^{\prime}, \nu^{\prime}\right)$ имеется в указанной теореме.

Напомним также, что если лог-оптимальный портфель по мере $Q$ существует, то $1 / \mathscr{E}\left(y^{*} L\right)$ есть решение задачи, двойственной к задаче максимизации логарифмической полезности по мере $Q$.

C помощью формулы (5) из теоремы 1 вытекает следующий результат.

Следствие 1. При $y=1$ решение $Y^{*}$ задачи (3), двойственной $\kappa$ задаче максимизачии степенной полезности (2), имеет вид

$$
Y^{*}=\frac{Z}{\mathscr{E}\left(y^{*} L\right)},
$$

где $Z$ - прочесс плотности меры $Q$ из теоремь 1 относительно меры $P$. $B$ частности, если прочесс $1 / \mathscr{E}\left(y^{*} L\right)$ является мартингалом по мере $Q$ и, следовательно, прочессом плотности некой меры относительно $Q$, то $Y^{*}$ есть прочесс плотности этой же меры относительно $P$.

Д о к а з а т е ль с т в о. Очертим план основной части доказательства теоремы 1.

1. Для $0<p<1$ из конечности $E\left(X_{T}^{*}\right)^{p}$ следует конечность $\int_{x>1} x^{p} d \nu$. Далее будем использовать именно это последнее предположение. Для $p<0$ математическое ожидание $E\left(X_{T}^{*}\right)^{p}$ конечно всегда.

2. Построение константы $y^{*}$. Доказательство того, что она однозначно определяется из триплета $L$.

3. Параметры Гирсанова $\beta=p y^{*}, Y=\left(1+y^{*} x\right)^{p}$ корректно определяют меру $Q$. Помимо этого, мы покажем, что $L$ является процессом Леви по мере $Q$ и задает эталонный портфель $\mathscr{E}\left(y^{*} L\right)$ по этой мере. Он же решает задачу степенной полезности для исходной меры $P$.

Сначала введем некоторые обозначения и отметим важные моменты общего характера. Зададим множество $\mathfrak{C}=\{p: \nu\{x: 1+p x<0\}=0\}$. Если у $L$ нет скачков, то $\mathfrak{C}$ есть вся прямая. Когда скачки ограничены, обозначим через $[\lambda, \gamma]$ минимальный отрезок (который может вырождаться в точку), содержащий $\operatorname{supp}(\nu)$. В случае наличия неограниченных скачков это будет полупрямая $[\lambda, \gamma), \gamma=+\infty$. Нетрудно проверить, что $\mathfrak{C}$ есть замыкание интервала $(\bar{M}, \bar{N})$, где

$$
\bar{M}=\left\{\begin{array}{ll}
-\frac{1}{\gamma} & \text { при } 0<\gamma<+\infty ; \\
-\infty & \text { при } \gamma \leqslant 0 ; \\
0 & \text { при } \gamma=+\infty ;
\end{array} \quad \bar{N}=\left\{\begin{aligned}
-\frac{1}{\lambda} & \text { при } \lambda<0 ; \\
+\infty & \text { при } \lambda \geqslant 0 ;
\end{aligned}\right.\right.
$$

$\bar{M}$ и $\bar{N}$ удовлетворяют соотношениям

$$
-\infty \leqslant \bar{M} \leqslant 0 \leqslant 1 \leqslant \bar{N} \leqslant+\infty .
$$

Хорошо известно (см. [5]), что немонотонность процесса $L$ эквивалентна всем стандартным условиям отсутствия арбитража в рассматриваемой экспоненциальной 
модели Леви, поэтому для задачи (2) о максимизации степенной полезности применима теорема 2.2 из статьи [12], согласно которой решение $X^{*}$ этой задачи существует и единственно. Более того, из пункта (ii) этой теоремы следует, что $X_{T}^{*}>0$ п.н., откуда $P\left(\inf _{t \leqslant T} X_{t}^{*}>0\right)=1$ ввиду отсутствия арбитража. Значит, в задаче $(2)$ достаточно рассматривать процессы $X$, удовлетворяющие $P\left(\inf _{t \leqslant T} X_{t}>0\right)=1$. Для таких $X$ удобнее пользоваться другим представлением. А именно, так как $X=1+\widetilde{H} \cdot S$, то, полагая $H=\left(\widetilde{H} S_{-}\right) / X_{-}$, получим

$$
1+X_{-} H \cdot L=1+\widetilde{H} S_{-} \cdot L=1+\widetilde{H} \cdot S=X .
$$

Значит, $X$ представим в виде

$$
X=\mathscr{E}(H \cdot L) .
$$

Верно и обратное: если $X$ представим в виде $(7)$ и $X>0, X_{-}>0$, то $X \in \mathscr{X}$. При этом $H_{t} \in \mathfrak{C} d P d t$-п.в. Доказательство этого факта можно найти в [5].

Следующая лемма включает в себя пункт 1 из плана доказательства.

Лемма 1. Для случая $0<p<1$ справедливы следующие утверждения.

1. Из условия $E\left(X_{T}^{*}\right)^{p}<\infty$ следует, ито

$$
\int_{x>1} x^{p} d \nu<\infty
$$

2. Если выполнено (8) и $X=\mathscr{E}(y L)$, где $y \in \mathfrak{C}$, то $E X_{T}^{p}<\infty$.

Д о к а з а т е л ь с т в о. Докажем первое утверждение леммы. Если скачки ограничены сверху, то конечность интеграла в (8) следует по определению меры Леви. В случае наличия неограниченных сверху скачков возьмем произвольное $y>0$ из $\mathfrak{C}$. Пусть процесс времени задается формулой $G_{t}=t$, а $\mu_{L}$ обозначает меру скачков процесса $L$. Преобразуем $\mathscr{E}(y L)^{p}$ с помощью формулы Ито при $0 \leqslant t \leqslant T$ :

$$
\begin{aligned}
& \mathscr{E}(y L)^{p}=\mathscr{E}\left(p y L+\frac{p(p-1)}{2} y^{2} c \cdot G+\left[(1+y x)^{p}-1-p y x\right] * \mu_{L}\right) \\
& =\mathscr{E}\left(p y b \cdot G+p y \cdot B+\frac{p(p-1)}{2} y^{2} c \cdot G+p y h *\left(\mu_{L}-\nu_{L}\right)\right. \\
& \left.+\left[(1+y x)^{p}-1-p y h\right] \mathbb{I}_{|x| \leqslant 1} * \mu_{L}+\left[(1+y x)^{p}-1\right] \mathbb{I}_{x>1} * \mu_{L}\right) \\
& =\mathscr{E}(D+A)=\mathscr{E}(D) \mathscr{E}(A),
\end{aligned}
$$

где $A_{t}=\left[(1+y x)^{p}-1\right] \mathbb{I}_{x>1} *\left(\mu_{L}\right)_{t}$, а $D_{t}$ есть сумма остальных слагаемых. Более того, процессы $A$ и $D$ независимы. Так как по предположению $E\left(X_{T}^{*}\right)^{p}<\infty$, то $E \mathscr{E}(A)_{T}<\infty$. Но $E \mathscr{E}(A)_{T}=E\left(1+\mathscr{E}\left(A_{-}\right) \cdot A_{T}\right) \geqslant E\left(1+A_{T}\right)$, откуда $E A_{T}<\infty$. Таким образом, процесс $\left[(1+y x)^{p}-1\right] \mathbb{I}_{x>1} * \mu_{L}$ интегрируем и имеет компенсатор $\left[(1+y x)^{p}-1\right] \mathbb{1}_{x>1} * \nu_{L}$. Отсюда следует сходимость интеграла $\int_{x>1}\left[(1+y x)^{p}-1\right] d \nu$, что эквивалентно конечности интеграла $\int_{x>1} x^{p} d \nu$.

Докажем второе утверждение леммы. Пусть конечен интеграл (8). Опять сделаем разложение по формуле Ито. В силу предположения процесс $A$ интегрируем. Так как мера Леви интегрирует $x^{2}$ в окрестности 0 , то процесс $\left[(1+y x)^{p}-1-p y h\right] \mathbb{I}_{|x| \leqslant 1} *$ $\left(\mu_{L}\right)_{t}$ также интегрируем. Добавляя и вычитая компенсаторы этих двух процессов, получаем:

$$
\mathscr{E}(y L)^{p}=\mathscr{E}\left(p y \cdot B+\left((1+y x)^{p}-1\right) *\left(\mu_{L}-\nu_{L}\right)+\varphi_{p}(y) \cdot G\right)=Z \exp \left(\varphi_{p}(y) \cdot G\right),
$$

где

$$
\varphi_{p}(y)=p y b+\frac{p(p-1)}{2} y^{2} c+\left[(1+y x)^{p}-1-p y h\right] * \nu .
$$


Нетрудно видеть, что согласно следствию 8.30 из [6] процесс $Z_{t}=\mathscr{E}\left(p y \cdot B+\left((1+y x)^{p}-\right.\right.$ $\left.1) *\left(\mu_{L}-\nu_{L}\right)\right)_{t}$ является равномерно интегрируемым мартингалом, следовательно, задает процесс плотности некоторой эквивалентной меры. Параметры Гирсанова при этом имеют вид $\beta=p y, Y(x)=(1+y x)^{p}$. Отсюда получаем $E \mathscr{E}(y L)_{T}^{p}=\exp \left(T \varphi_{p}(y)\right)<$ $\infty$, что и требовалось показать. Лемма 1 доказана.

Разложение Ито, которое мы сделали в лемме для некоторой константы $y \in \mathfrak{C}$, можно точно так же провести и для случая $p<0$ с той лишь оговоркой, что $y L$ не должно иметь скачков величины -1 с положительной вероятностью. Последнее может случиться лишь в граничных точках $\mathfrak{C}$, и тогда $E U\left(\mathscr{E}(y L)_{T}\right)=-\infty$, а значит, $y$ заведомо не определяет эталонный портфель.

Начиная с этого момента предполагаем, что выполнено условие (8).

Теперь приведем некоторые наводящие рассуждения, объясняющие выбор меры $Q$ и построение константы $y^{*}$. Основная часть этих выкладок будет использована в пункте 3 доказательства.

Запишем известное неравенство для вогнутых функций, примененное к $U(x)=$ $x^{p} / p$ и случайным величинам $X_{T}^{*}, X_{T}$, где $X, X^{*} \in \mathscr{X}$ строго положительны:

$$
U\left(X_{T}\right) \leqslant U\left(X_{T}^{*}\right)+\left(X_{T}-X_{T}^{*}\right) U^{\prime}\left(X_{T}^{*}\right) .
$$

Из этой формулы видно, что если $E_{P}\left(X-X^{*}\right) U^{\prime}\left(X^{*}\right) \leqslant 0$ для любого $X \in \mathscr{X}$, то $X^{*}$ будет оптимальным портфелем для меры $P$. Преобразуем данное слагаемое, предполагая, что $X^{*}=\mathscr{E}\left(y^{*} L\right)$ для некоторой константы $y^{*}$ :

$$
\left(X_{T}-X_{T}^{*}\right) U^{\prime}\left(X_{T}^{*}\right)=\left(X_{T}-X_{T}^{*}\right)\left(X_{T}^{*}\right)^{p-1}=\left(\frac{X_{T}}{X_{T}^{*}}-1\right) \mathscr{E}\left(y^{*} L\right)_{T}^{p} .
$$

Как мы убедились в доказательстве второго утверждения леммы $1, \mathscr{E}\left(y^{*} L\right)_{t}^{p}=$ $Z_{t} \mathscr{E}\left(t \varphi_{p}\left(y^{*}\right)\right)$, где $Z_{t}$ задает процесс плотности перехода к новой мере с параметрами Гирсанова $\beta=p y^{*}, Y=\left(1+y^{*} x\right)^{p}$. Если $\mathscr{E}\left(y^{*} L\right)$ задает эталонный портфель относительно меры $Q$, то

$$
E_{P}\left(\frac{X_{T}}{X_{T}^{*}}-1\right) Z_{T} \exp \left(T \varphi_{p}\left(y^{*}\right)\right)=\exp \left(T \varphi_{p}\left(y^{*}\right)\right) E_{Q}\left(\frac{X_{T}}{X_{T}^{*}}-1\right) \leqslant 0 .
$$

Таким образом, если константа $y^{*}$ такова, что $\mathscr{E}\left(y^{*} L\right)$ есть эталонный портфель относительно меры $Q$, задаваемой указанными параметрами Гирсанова (зависящими от $y^{*}$ ), то этот же портфель будет оптимальным в исходной задаче максимизации степенной полезности.

Теперь займемся пунктом 2 доказательства - поиском требуемой константы $y^{*}$. Триплет $\left(b^{\prime}, c^{\prime}, \nu^{\prime}\right)$ по предполагаемой мере $Q$ пересчитывается через исходный триплет по формулам (6). Это позволяет записать функцию $F$ из [5], определенную на $\mathfrak{C}$, чьи свойства использовались для решения задачи (2) в логарифмическом случае:

$$
F(y)=-b-c p y^{*}+c y+\int\left(h(x)-\frac{x\left(1+y^{*} x\right)^{p}}{1+y x}\right) \nu(d x) .
$$

Для нее в полной мере применимы результаты из упомянутой работы. Однако пользоваться ею сложно, так как она зависит от $y$ и $y^{*}$. Нас главным образом интересует поведение $F$ в точке $y^{*}$, поэтому объединим эти переменные и введем функцию

$$
F_{p}(y)=-b+c(1-p) y+\int\left(h(x)-\frac{x}{(1+y x)^{1-p}}\right) \nu(d x) .
$$

Аналогичная функция использовалась в работе [9]. Как и $F$, функция $F_{p}$ определена в $\mathfrak{C}$. В следующей лемме мы покажем, что $F_{p}$ корректно определена, а значения $+\infty$ и $-\infty$ может принимать только в граничных точках $\mathfrak{C}$. 
Лемма 2. Функиия $F_{p}(y)$ корректна определена, непрерывна и монотонно возрастает на $\mathfrak{C}$, а также конечна в интервале $(\bar{M}, \bar{N})$.

Д о к а з а т е л ь с т в о во многом аналогично доказательству в логарифмическом случае, с той лишь оговоркой, что надо использовать условие (8).

Понятно, что слагаемое $-b+c(1-p) y$ непрерывно и строго монотонно возрастает на $(\bar{M}, \bar{N})$. Оставшийся интеграл представим в виде разности двух составляющих:

$$
\begin{aligned}
& I_{1}(y)=\int_{|x| \leqslant 1} x\left(1-\frac{1}{(1+y x)^{1-p}}\right) \nu(d x)=\int_{|x| \leqslant 1} \frac{x\left((1+y x)^{1-p}-1\right)}{(1+y x)^{1-p}} \nu(d x), \\
& I_{2}(y)=\int_{x>1} \frac{x}{(1+y x)^{1-p}} \nu(d x) .
\end{aligned}
$$

Напомним, что из определения процесса Леви следует, что выполнены соотношения

$$
\nu\{0\}=0, \quad \int\left(x^{2} \wedge 1\right) \nu(d x)<\infty .
$$

Рассмотрим интеграл $I_{1}$. Из определения $\mathfrak{C}, \bar{M}, \bar{N}$ следует, что для любого $y \in(\bar{M}, \bar{N})$ и любого $x \in \operatorname{supp}(\nu)$ выполнено $(1+x y)^{1-p} \geqslant \epsilon_{0}(y)>0$, т.е. при $x \in \operatorname{supp}(\nu)$ знаменатель подынтегрального выражения ограничен снизу. Числитель же ограничен по модулю функцией $K(y) x^{2}$, где $K(y)>0$ есть некоторая константа. Отсюда и из (14) следует конечность $I_{1}$ для любого $y \in(\bar{M}, \bar{N})$.

Теперь докажем конечность $I_{2}$. Если $y>0$, то для достаточно больших $x$ подынтегральное выражение будет эквивалентно $y^{p-1} x^{p}$. Из (8) следует сходимость этого выражения. Пусть $y \leqslant 0$. При $\bar{M}<0$ числитель подынтегрального выражения в $I_{2}$ ограничен $\gamma<\infty$. Знаменатель ограничен в силу рассуждений, проведенных для $I_{1}$. Если $\bar{M}=0$, то $y$ не лежит в $(\bar{M}, \bar{N})$.

Таким образом, мы показали, что $I_{1}$ и $I_{2}$ определены и конечны для любого $y \in(\bar{M}, \bar{N})$. Нетрудно убедиться в том, что при возрастании $y$ подынтегральное выражение в $I_{1}$ не убывает, а в $I_{2}$ не возрастает. Поэтому по теореме о монотонной сходимости интегралы $I_{1}$ и $I_{2}$ будут непрерывны и монотонны по $y$ на интервале $(\bar{M}, \bar{N})$. Отсюда заключаем, что $F_{p}(y)$ конечна, непрерывна и монотонно возрастает по $y$ в $(\bar{M}, \bar{N})$. Более того, $F_{p}$ строго возрастает в $(\bar{M}, \bar{N})$, так как в противном случае получаем, что $\nu=0$ и $c=0$, откуда $b=0$, но тождественно нулевой процесс $L$ мы исключили из рассмотрения. Из теоремы о монотонной сходимости также вытекает существование пределов, возможно и бесконечных, в $\bar{M}$ и $\bar{N}$.

Покажем, что $F_{p}$ корректно определена в граничных точках. Действительно, в силу возрастания каждый из интегралов $I_{1}$ и $-I_{2}$ в точке $\bar{N}$ либо конечен, либо расходится к $+\infty$, а в $\bar{M}$ либо конечен, либо расходится к $-\infty$. Отсюда следует требуемое. Лемма 2 доказана.

С помощью этой леммы легко проверить, что для триплета, отвечающего немонотонному процессу Леви и удовлетворяющего условию (8), существует единственное $y^{*}$, для которого выполнено ровно одно из следующих утверждений:

$-y^{*}$ есть корень уравнения $F_{p}(y)=0$;

$-y^{*}=\bar{M}, \bar{M}>-\infty$ и $+\infty>F_{p}\left(y^{*}\right)>0$;

$-y^{*}=\bar{N}, \bar{N}<+\infty$ и $-\infty<F_{p}\left(y^{*}\right)<0$.

Действительно, при отсутствии скачков или конечности $\bar{M}$ и $\bar{N}$ это следует из строгой монотонности $F_{p}$. Пусть $\bar{M}=-\infty, \bar{N}<+\infty$. Если упомянутые выше соотношения не выполнены, то $F_{p}>0$ в $\mathfrak{C}$. Заметим, что равенство $\bar{M}=-\infty$ означает отсутствие положительных скачков, и тогда предел при $y \rightarrow-\infty$ интеграла в (13) равен $\int x \mathbb{I}_{x<0} \nu(d x)$. Поэтому положительность функции $F_{p}$ на $\mathfrak{C}$ влечет за собой соотношения $c=0$ и $-b+\int x \mathbb{I}_{x<0} \nu(d x) \geqslant 0$. Последнее неравенство означает, что процесс $L$ монотонный. Случай $\bar{M}>-\infty, \bar{N}=+\infty$ разбирается аналогично. 
Покажем, что предложенный вариант константы $y^{*}$ есть именно то, что требуется в пункте 3 доказательства. Проведя рассуждения, аналогичные выкладкам во второй части леммы 1 , получим, что параметры Гирсанова $\beta=p y^{*}, Y(x)=\left(1+y^{*} x\right)^{p}$ задают меру $Q$ и $E \mathscr{E}\left(X^{*}\right)_{t}^{p}=\exp \left(t \varphi_{p}\left(y^{*}\right)\right) E Z_{t}<\infty$, где $\varphi$ есть детерминированная функция. Отметим, что мера $Q$ эквивалентна $P$. Для этого достаточно проверить, что $\nu\left\{1+y^{*} x=0\right\}=0$. Если $y^{*}$ лежит внутри $\mathfrak{C}$, то это очевидно. Если же $y^{*}=\bar{M}$ или $y^{*}=\bar{N}$, то предположение $\nu\left\{1+y^{*} x=0\right\}>0$ ведет к расходимости интеграла в определении $F_{p}$. Новый триплет (6) является детерминированным, откуда следует, что по мере $Q$ процесс $L$ останется процессом Леви. Поскольку меры $P$ и $Q$ эквивалентны, то $L$ относительно $Q$ будет также немонотонным. Это, впрочем, легко вывести из вида триплета (6). Нетрудно понять, что если $F_{p}$ в точке $y^{*}$ удовлетворяет какому-то из трех вышеупомянутых условий, то для функции $F$ это условие также выполнено, отсюда в силу результатов статьи [5] следует, что данная константа $y^{*}$ определяет решение для задачи логарифмической полезности по мере $Q$. Осталось показать, что $\mathscr{E}\left(y^{*} L\right)$ решает и исходную задачу степенной полезности. Для построенной меры $Q$ мы снова можем записать соотношения (10)-(12). Так как $E \mathscr{E}\left(X^{*}\right)_{T}^{p}=\exp \left(T \varphi_{p}\left(y^{*}\right)\right) E Z_{T}<\infty$, то математические ожидания обоих слагаемых в правой части (10) конечны. Это означает, что в (10) и (11) мы можем добавить знаки математического ожидания. Применим (12) к (10), отсюда и следует требуемое. Пункт 3 доказательства завершен.

Из наших рассуждений с учетом леммы 1 следует эквивалентность условий (8) и $E\left(X_{T}^{*}\right)^{p}<\infty$. Это завершает доказательство теоремы 1.

Д о к а з а т е ль с т в о т е о р е м ы 2. Рассмотрим отображение, которое триплету $(b, c, \nu)$ из $\mathscr{Q}_{p}$ ставит в соответствие триплет $\left(b^{\prime}, c^{\prime}, \nu^{\prime}\right)$ из $\mathscr{Q}$ по формулам $(6)$ с константой $y^{*}$, определяемой в теореме 1 . Нам нужно показать, что у каждого элемента из $\mathscr{Q}$ есть ровно один прообраз из $\mathscr{Q}_{p}$.

Пусть у нас есть триплет $\left(b^{\prime}, c^{\prime}, \nu^{\prime}\right)$ из $\mathscr{Q}$. В работе [5] было доказано, что существует единственная константа $z^{*}$, для которой $\mathscr{E}\left(z^{*} L\right)$ есть эталонный портфель по мере $Q$. Рассмотрим меру $P$, которая получается из $Q$ преобразованием с параметрами Гирсанова $\left(\beta^{\prime}, Y^{\prime}\right)=\left(-p z^{*},\left(1+x z^{*}\right)^{-p}\right)$. В силу уже упоминавшегося следствия 8.30 из [6] она существует и по ней триплет характеристик процесса $L$ является детерминированным и однородным, т.е. имеет вид (4) с некоторыми $(b, c, \nu)$. Пользуясь явным видом для $(b, c, \nu)$ из теоремы III.3.24 в [7], нетрудно убедиться, что триплет $(b, c, \nu)$ принадлежит $\mathscr{Q}_{p}$ и для него константа $y^{*}$ из теоремы 1 совпадает с $z^{*}$. Значит, образом меры $P$ при рассматриваемом отображении является $Q$. Если для произвольной меры $P$ мы построим меру $Q$ как в теореме 1 , то для нее $y^{*}$ определяет эталонный портфель, т.е. совпадает с $z^{*}$. Отсюда следует единственность прообраза. Теорема 2 доказана.

Автор выражает благодарность научному руководителю А.А.Гущину за поддержку и полезные советы, а также рецензенту за ряд ценных замечаний.

\section{СПИСОК ЛИТЕРАТУРЫ}

1. Delbaen F., Schachermayer $W$. The fundamental theorem of asset pricing for unbounded stochastic processes. - Math. Ann., 1998, v. 312, № 2, p. 215-250.

2. Esche F., Schweizer M. Minimal entropy preserves the Lévy property: how and why. Stochastic Process. Appl., 2005, v. 115, № 2, p. 299-327.

3. Goll T., Kallsen J. A complete explicit solution to the log-optimal portfolio problem. - Ann. Appl. Probab., 2003, v. 13, № 2, p. 774-799.

4. Goll T., Rüschendorf L. Minimax and minimal distance martingale measures and their relationship to portfolio optimization. - Finance Stoch., 2001, v. 5, № 4, p. 557-581. 
5. Иванов М. Ю. Максимизация логарифмической полезности в экспоненциальной модели Леви. - Вестн. Моск. ун-та, 2014, № 6, с. 16-24.

6. Jacod J. Calcul Stochastique et Problèmes de Martingales. Berlin-Heidelberg-New York: Springer-Verlag, 1979, 539 p.

7. Jacod J., Shiryaev A.N. Limit Theorems for Stochastic Processes. Berlin: Springer, 2003, $661 \mathrm{p}$.

8. Jeanblanc M., Klöppel S., Miyahara $Y$. Minimal $f^{q}$-martingale measures for exponential Lévy processes. - Ann. Appl. Probab., 2007, v. 17, № 5-6, p. 1615-1638.

9. Kallsen J. Optimal portfolios for exponential Lévy processes. - Math. Methods Oper. Res., 2000, v. 51, p. 357-374.

10. Karatzas I., Lehoczky J. P., Shreve S. E. Optimal portfolio and consumption decisions for a «small investor» on a finite horizon. - SIAM J. Control Optim., 1987, v. 25, p. $1557-1586$.

11. Kardaras $C$. No-free-lunch equivalences for exponential Lévy models under convex constraints on investment. - Math. Finance, 2009, v. 19, № 2, p. 161-187.

12. Kramkov D., Schachermayer $W$. The asymptotic elasticity of utility functions and optimal investment in incomplete markets. - Ann. Appl. Probab., 1999, v. 9, №3, p. $904-950$.

13. Larsen $K$. A note on the existence of the power investors optimizer. - Finance Stoch., 2011, v. 15, № 1, p. 183-190.

14. Nutz M. Power utility maximization in constrained exponential Lévy models. - Math. Finance, 2012, v. 22, № 4, p. 690-709.

15. Sato K.-I. Lévy Processes and Infinitely Divisible Distributions. Cambridge: Cambridge Univ. Press, 2003, 486 p.

16. Takaoka K., Schweizer M. A note on the condition of no unbounded profit with bounded risk. — Finance Stoch., 2014, v. 18, № 3, p. 393-405.

Поступила в редакцию 22.VIII.2013

Исправленный вариант

9.X.2014

(C) 2014 г. $\quad$ AYDOGMUS O.*, GHOSH A.P.**, COLORED MAXIMAL BRANCHING PROCESS

\begin{abstract}
Рассматривается версия многотипного максимального ветвящегося процесса, введенного недавно А.В.Лебедевым. Основным результатом работы является предельная теорема для эмпирических частот типов. Показано, как изменяется со временем первоначальное распределение типов под воздействием механизма селекции среди соревнующихся индивидуумов максимального ветвящегося процесса.
\end{abstract}

Ключевые слова и фразы: многотипные максимальный ветвящийся процесс, максимальный ветвящийся процесс, асимптотическое поведение цепей Маркова, аддитивные функционалы на цепях Маркова.

* Department of Mathematics, Iowa State University, Ames, IA 50011; e-mail: aydogmus@iastate.edu

** Departments of Statistics and Mathematics, Iowa State University; e-mail: apghosh@iastate.edu

*** Departments of Statistics and Mathematics, Iowa State University; e-mail: sghosh@iastate.edu

† Department of Mathematics, Iowa State University; e-mail: roiterst@iastate.edu 